\title{
Anstelle eines Editorials: 'Modell Deutschland' in der Diskussion
}

Im folgenden veröffentlichen wir das Protokoll einer Arbeitskonferenz der PROKLA-Redaktion zum Thema 'Modell Deutschland'. Da wir meinen, daß auf dieser Arbeitskonferenz nicht nur wichtige Aspekte der Diskussion um das 'Modell Deutschland' herausgearbeitet werden konnten, sondern auch die Schwierigkeiten mit dem Begriff und den vorliegenden Analysen deutlich wurden, ist die Darstellung dieser Diskussion eine geeignete Einleitung für den vorliegenden zweiten Diskussionsband zum 'Modell Deutschland' sozusagen als Nach- und Vorbereitung.

$\mathrm{Zu}$ der Arbeitskonferenz wurden alle Mitarbeiter der Hefte 40 und 41 eingeladen, als Vorbereitung dienten die in Heft 40 veröffentlichten Beiträge.

\section{Arbeitskonferenz vom 29.9.1980:}

Was ist eigentlich das 'Modell Deutschland'?

Steht dieser Begriff stellvertretend für die sozialliberale Reformpolitik (»Innere Reformen«), dann kann man das Scheitern dieses Modells mit der Krise 1974/75 und der monetären Beschneidung vieler Reformvorhaben ansetzen und daraus die Notwendigkeit einer linken Alternative ableiten. Nimmt man dagegen den Wahlkampfslogan der SPD zu den Wahlen 1976 beim Wort, dann scheint damit offensichtlich nicht (nur) die begrenzte Reformphase gemeint zu sein, sondern eine in Prosperität und Krise vorhandene spezifische Konfliktverarbeitung durch die Gesellschaft, in deren Zentrum die Integration der Gewerkschaften resp. der Arbeiterschaft im weiteren Sinne in das politische System steht. Daraus ergeben sich erhebliche Konsequenzen für Ansatzpunkte linker alternativer Politik. Damit weisen aber zugleich die Grundlagen dieses Modells gesellschaftlicher Konfliktverarbeitung hinter die sozialliberale und auch die Große Koalition zurück. In den Beiträgen zu Heft 40 wurde dieser Begriff entsprechend unterschiedlich - und verwirrend - verwendet: als quasi regierungs-amtliche Programmatik, deren Scheitern sodann konstatiert wurde (SOST), als spezifische Form der Weltmarkteinbindung des deutschen Kapitals mit daraus sich ableitender Spaltung der Arbeiterbewegung (KernRand-Esser/Fach/Simonis) oder als spezifisch deutsche Form der Integration der Arbeiterschaft in politische bzw. gesellschaftliche Lösungsversuche von kapitalistisch erzeugten Konflikten, die auf den historischen korporativen Strukturen der deutschen Gesellschaft aufbauen können (Editorial Nr. 40).

\section{'Modell Deutschland' als Form gesellschaftlicher Konfliktlösungspolitik}

Wird der Akzent beim 'Modell Deutschland' auf die spezifische Politikform der Vermittlung gesellschaftlicher Konflikte im Gefolge der Krise und Stagnationsphase seit 1975 gelegt, dann wird der Formwandel der staatlichen Wirtschaftspolitik mit seinen gesellschaftspolitischen Konsequenzen bedeutsam: Die Wirtschaftspolitik betreibt nicht mehr nur »Niveauaussteuerung«, sondern will die öko- 
nomischen Tendenzen verstärken, die sich als Konsequenz von Krise, Stagnation und Konkurrenz auf dem Weltmarkt ergeben haben. In dem Konzept von Scharpf / Hauff wird dies am deutlichsten gefaßt (auch wenn die von ihnen geforderte »aktive Strukturpolitik« noch weitgehend Konzeption geblieben ist). Wichtig ist hierbei, daß die Veränderung der vorherrschenden staatlichen Wirtschaftspolitik von einer eher gesamtwirtschaftlich-quantitativ orientierten zu einer branchenorientierten Struktur-und Technologiepolitik nicht nur die Einzelgewerkschaften verstärkt in korporativistische Interessensvertretungspolitiken drängt (oder sie darin bestärkt). Darüberhinaus erhebt sich auch die Frage, ob nicht eine solche Politik ihre gesellschaftliche Basis und ihr Ziel - die Absicherung des Kerns der Belegschaften und Gewerkschaftsmitglieder - untergräbt, weil sie weltmarktorientiert auf die Modernisierung von Wachstumsbranchen setzen muß. Das aber heißt u.U. nicht nur »Gesundschrumpfen« von strukturschwachen Branchen (mit entsprechenden Arbeitsplatzverlusten), sondern auch Rationalisierung und damit Dequalifikation, Arbeitsintensivierung und Bedrohung der angestammten Arbeitsplätze bei Facharbeitern der Wachstumsbranchen.

Gleichzeitig wurde in der Diskussion die Festigkeit des hinter dieser Wirtschafts- (und Sozial-) politik stehenden 'sozialen Konsens' betont, der dort, wo er zerbröckelt, aufgrund der Individualisierung und Partialisierung von Krisenerfahrungen und Diffundierung des gesellschaftlichen Konfliktpotentials kaum bedroht wird. Bei der 'Modell Deutschland' spezifischen Form gesellschaftspolitischer Konfliktlösungspolitik handelt es sich nicht um die Differenz des SPD- zum CDU-Staat, sondern die Unterschiede müssen innerhalb des SPD-Staats selbst gesehen werden. Und zwar an dem Übergang zu den veränderten gesellschaftlichen Strukturen nach dem 73-75er Einbruch, an dem sich dieser andere Strategietypus von Regierungspolitik und Loyalitätsproduktion festmacht. D.h. auch, daß sich heute die Bindungen der Gewerkschaften an die SPD nicht mehr so sehr an Reformhoffnungen festmachen, sondern daran, daß der SPD die höchste Krisenbewältigungskapazität zugemessen wird. In der Diskussion stellte sich dabei die Frage, inwieweit es sich bei den so charakterisierten Prozessen 1. um 'neue' Momente handelt, oder ob nicht vielmehr diese heute befürchtete Produktion von Entsolidarisierung und Konkurrenz z.B. in der Sozialpolitik immer schon gegeben ist, ja im Grunde ihr Kernprinzip darstellt, das demzufolge auch weder durch eine CDU- noch eine SPD-Regierung in seinen Grundelementen je angetastet worden ist. 2. inwiefern dies überhaupt als eine bewußte Strategie bezeichnet werden kann oder konkreter: Die Frage inwieweit die gegebene Gesellschaftssituation von einer bestimmten Partei, von sozialdemokratischer Politik gemacht, gesteuert wird oder nicht. So ist zum Beispiel die 'Marginalisierung' nicht so sehr politisch 'gemacht', sondern von der Ökonomie produziert worden. Denn die Betriebe haben heute Formen der Beschäftigungspolitik entwickelt, die eben genau auf das abzielen, was als 'Kern' und 'Rand' innerhalb der Beschäftigten bezeichnet wurde. Die damit einhergehende Einbindung der Gewerkschaften in diese Betriebs- und Branchenpolitik der Kapitale, die Verstärkung korporativistischer Politikelemente bei den Gewerkschaften werden zwar auf gesamtgesellschaftlicher Ebene wieder aufgenommen. Bei Scharpf / Hauff in Form der konzentrierten Aktion, die im Unterschied zur konzertierten Aktion nicht mehr gesamtgesellschaftlich auf die Solidarität aller Arbeitnehmer aufbaut, sondern sich nur noch auf Branchen bezieht und die auseinandertreibenden Entwicklungsmomente auf Betriebsebene aufgreift und zementiert. Dabei wäre aber zu fragen, ob das zugrundeliegende Prinzip, nämlich die Anpassung der Kapitalstrukturen an die Weltmarkterfordernisse als einem Kernmechanismus des 'Modell Deutschland' und die darauf aufbauenden Politiken, z.B. der Marginalisierung, nicht auch unter einer CDU-Regierung wirken würde oder jedenfalls nicht wesentlich veränderbar ist. »Der politische Einfluß ist gering, gesteuert werden diese Strategien von Konzernpolitik« (U. Jürgens).

Gegenüber dieser Betonung der Krisenpolitik mit ihren gesellschaftlichen Konsequenzen wurde von J. Hirsch der umfassende gesellschaftliche Restrukturierungsprozeß, der sich auch im Begriff des 'Modell Deutschland' ausdrückt, hervorgehoben: „Die ganze Organisationsform der Gesellschaft verändert sich, die Bedeutung von Gewerkschaften und Arbeitgeberverbänden wird sich wahtscheinlich im Zuge dieses Restrukturierungsprozesses erheblich verschieben, spezifische Privilegienstrukturen, die sich gar nicht in den traditionellen Klassenschemata abbilden lassen, werden sich verändern (Mittelklassen)«. Auch hinter dem Begriff 'neuer Akkumulationstyp' verbergen sich Prozesse, an denen 
deutlich wird, in welch gravierendem Umfang Kapitalakkumulation gesellschaftliche Desorganisation und Reorganisation bedeutet. Die Weltmarktstrategien, wie sie sich z.B. im 'Modell Deutschland' entwickeln, sind nicht so sehr Expansionsstrategien des Kapitals auf gleichbleibender gesellschaftlicher Ebene, sondern es geht dabei darum, gesellschaftliche Strukturveränderungen mehr oder weniger elegant durchzusetzen. Wenn also Sicherung des Profits heißt: forcierte Veränderung gesellschaftlicher Strukturen, dann heißt das zugleich eine veränderte Rolle des Staates. Ein solches spezifisches Einbeziehen des Staates in den gesellschaftlichen Reproduktionszusammenhang läßt dann die Grenze zwischen ökonomischer Entwicklung und politischer Strategie fragwürdig werden. Denn es könnte sich herhausstellen, daß die Art und Weise, wie eine ökonomische Krise zur gesellschaftlichen Krise wird, von der Wirkung politischer Prozesse abhängt, die sie vorantreibt oder auch nicht vorantreibt. Die Frage von Krisen, Konflikten und Bruchstellen läßt sich dann nicht auf der Ebene politischer Strategien, sondern nur auf der Ebene von politischen Prozessen, deren Grundlage natürlich die Bewegung des Kapitals darstellt, festmachen.

Eine ganz andere Einschätzung sowohl des Begriffs 'Modell Deutschland' als auch der aktuellen Krisenbewältigungskapazitäten der SPD-Regierung wurde von SOST vertreten: Aufgrund des internationalen Zurückgehens der Expansion der Wachstumsraten geht es auf dem Weltmarkt darum, durch verstärkte Konkurtenz und dem Ausspielen von Produktivitätsvorteilen Weltmarktanteile auf Kosten anderer Konkurrenten zu gewinnen. Diese Offensive wird zu Importrestriktionen und protektionistischen Tendenzen führen, die das exportabhängige BRD-Kapital empfindlich treffen müssen. »Ohne wieder die Totenglocke läuten zu wollen, gehen wir daher doch mittelfristig von einer Verschärfung der ökonomischen -und damit auch der sozialen Widersprüche aus, und zwar nicht nur aufgrund der Weltmarktzusammenhänge, sondern auch, weil das, was als klassisch sozialdemokratische Politik bezeichnet werden kann, gescheitert ist. Deshalb sprechen wir auch vom Scheitern des 'Modell Deutschland'«. »Die Strategie der Sozialdemokratie ist ein planloses Löcherstopfen, eine Umverteilung von Lasten, und nicht mehr des ökonomischen Zuwachses, sie ist eben gerade kein offensives Modell, sondern ein perspektivloses Zurückweichen vor unkontrollierbaren Prozessen und der Zuspitzung von Konflikten.»

\section{'Fordismus' und 'neue soziale Bewegung' - Eine Forschungshypothese}

In einem zweiten Teil der Diskussion wurde auf die aus der Einschätzung des 'Modell Deutschland' resultierenden Konsequenzen, Schwierigkeiten und Möglichkeiten für linke Politik eingegangen, wobei sich die Debatte im wesentlichen auf die 'neue soziale Bewegung' und den von Hirsch / Roth aufgeworfenen Begriff des 'Fordismus' konzentrierte.

Auf der einen Seite kann festgehalten werden, daß auch innerhalb einer Gesellschaftsformation, ohne ihre Grundgesetze außer Kraft zu setzen, doch sehr wesentliche und entscheidende Veränderungen stattfinden, die nachhaltige Auswirkungen auf Klassenstrukturen, den gesellschaftlichen Reproduktionszusammenhang und das Verhältnis von Staat und Gesellschaft haben. Doch fragt sich, ob man dies mit dem Begriff 'Fordismus' erfassen kann. Hirsch / Roth selbst wollen ihren Ansatz primär als eine Forschungshypothese verstanden wissen, die sich aus dem Umstand motiviert, daß von marxistischer Seite aus vorliegenden Theorieansätzen zu diesem Problem (von Modernisierungstheorien bis zur Frankfurter Schule) entweder die Stamokaptheorie oder das Beharren auf der Gültigkeit der Akkumulationsgesetzlichkeit entgegengehalten wurden. Die marxistische Linke hat sich also dem Problem nicht selbst gestellt. „Der Vergesellschaftungsbegriff ist ein schwammiger, ein Begriff, der auf ein Defizit in der Theoriebildung verweist - angefangen bei Marx selbst. Marx hat die notwendige Tendenz des Kapitals zur reellen Subsumtion der Arbeitskraft und aller sozialen Sphären konstatiert, sich aber nie Gedanken darüber gemacht, was das eigentlich heißt. Grundsätzlich werden mit der Ausdehnung der Mehrwertproduktion auch immer mehr Bereiche der Reproduktionssphäre z.B. der unmittelbaren Subsistenzwirtschaft entzogen und der Marktwirtschaft unterworfen. D.h., bestimmten Entwicklungsstufen im Produktionsbereich korrespondieren entsprechende Entwicklungen im Re- 
produktionsbereich, wofür der Begriff 'Fordismus' analog zum Taylorismus ein Bild sein sollte. Die historische Präzisierung dieser Prozesse ist damit noch nicht hinreichend erfaßt, wichtiger scheinen auch die Fragen nach den hieraus resultierenden Konsequenzen: So wie beispielsweise durch den Taylorismus die Arbeiter ihrer Qualifikationen und Kompetenzen enteignet werden, so finden auch im Reproduktionsbereich Veränderungen sozialer Strukturen, gesellschaftliche Desintegrationstendenzen statt, woraus sich die Frage nach den Handlungskompetenzen des Proletariats ergibt, d.h. die Frage, wie diese Kompetenzen, die das Proletariat zum revolutionären Subjekt machen, angesichts der beschriebenen Prozesse eigentlich noch zustande kommen können.« (J. Hirsch)

Der Mangel des Begriffs 'Fordismus' blieb auch ein Mangel in der Diskussion: Man war sich einig in Bezug auf die Tragweite und Bedeutung historischer, gesellschaftspolitischer Umstrukturierungsprozesse, beispielsweise der Durchdringung des gesellschaftlichen Lebens mittels zentraler Kommunikation, oder der heute möglichen Erfahrung von Subjektivität, die erst aufgrund einer durch die Sozialpolitik ermöglichten individuellen Lebensplanung überhaupt realisierbar ist; aber man war sich nicht einig darin, wie diese historisch mehr oder weniger kontinuierlichen Veränderungsprozesse in ihrer Qualität theoretisch faßbar sind. Gibt es bei solchen Prozessen wie 'Durchstaatlichung', 'Durchkapitalisierung' einen Endpunkt, Umschlagspunkt oder dergl., die der Begriff 'Fordismus' bezeichnen soll, in ihrer Qualität bisher aber keineswegs ausreichend erfaßt hat? So berechtigt die Hirsch / RothBetonung der Prozesse sozialer Desintegration, der Zerstörung 'proletarischen Milieus' auch in Kernbereichen und der Unmöglichkeit, innerhalb des Systems biographische Lebensperspektiven zu entwerfen, sein mag, so läßt doch die Stoßrichtung der Fragestellung den ambivalenten Charakter der Formen, der Vergesellschaftungsform außer Acht.

Sowohl in dem Begriff 'Fordismus' als auch in der Vokabel 'soziale Desintegration' sind keine Elemente davon enthalten, wie das, was bürgerlich kapitalistisch erzeugt worden ist, Freiheit, Demokratie und Subjektivität ambivalent ist. Wenn diese Ambivalenz nicht auch theoretisch expliziert wird, ist man an keiner Stelle in der Lage, positive Perspektiven zu entwickeln. Und so liegt auch die Schwierigkeit, sich klarzumachen, in welche Richtung die 'neue soziale Bewegung' geht, methodisch darin, daß der ambivalente Charakter der Formen (in dem Artikel von H/R) nicht untersucht worden ist.

In der hier protokollierten Diskussion sind eine Reihe von Fragen aufgeworfen und kontrovers im Raum stehen gelassen worden. Wir wollen diese Diskussion an einzelnen Inhalten weiterverfolgen und damit auch der Gefahr aus dem Wege gehen, durch die mit dem Begriff 'Modell Deutschland' vorgegebene Vielfalt an Fragen und Problemen in zu vage Globalbetrachtungen zu verfallen. So ist beispielsweise Heft 42 mit dem Schwerpunkt 'Weltmarkt' geplant, von dessen Zusammenhängen her ja auch das 'Modell Deutschland' begründet wird: Heft 43 beschäftigt sich vor allem mit der 'neuen sozialen Bewegung', und auch das vorliegende Heft knüpft an einige der im Protokoll bezeichneten Probleme an:

- Das 'Modell Deutschland' in der Sicht des Auslands (mit Artikeln von Markovits/Ertman -USA, Weill/Frettchen und Adler - Frankreich, Bobbio, Bolaffi / Marramao Italien) thematisiert nochmals die Frage nach den spezifischen Bestandteilen sozialdemokratischer Politik im Rahmen der aktuell praktizierten Krisenlösungsstrategie, der ökonomischen Situation der Bundesrepublik im Weltmarktzusammenhang und vor allem die veränderten Entwicklungsbedingungen für gewerkschaftliche Politik und das Verhältnis von Arbeiterbewegung und neuer sozialer Bewegung. Spannend ist dabei die je nach Land variierende Sichtweise des 'Modell Deutschland', seine offizielle Rezeption und die daran entwickelte sozialistische Kritik. Steht dies einerseits im Diskussionszusammenhang des jeweiligen Landes (über den wir somit etwas lernen), so verdeutlicht es uns auf der anderen Seite sowohl die Relativität einzelner Problempunkte, als auch die über die BRD hinausweisende Bedeutung der bei uns ablaufen- 
den politischen Prozesse und Diskussionen (was da und dort auch wieder Mut machen kann).

- Hübner/Stanger setzen sich mit den von den Sozialistischen Studiengruppen entwickelten Alternativen der Wirtschaftspolitik auseinander, überprüfen deren politische Grundlagen und politischen Folgerungen.

- Jäger knüpft in seinem Diskussionsbeitrag an die in der PROKLA (Heft 38) begonnene Korporatismus-Debatte an und versucht zu begründen, daß man Korporatismus nur als einen parteienspezifischen begreifen kann.

- Die unter Dokumentation abgedruckten Artikel von E. Maire und André Gorz sollen einen Beitrag zur Diskussion um die Entwicklung sozialistischer Strategien und das Verhältnis von Arbeiter- und neuer sozialer Bewegung liefern.

Die Redaktion

Angesichts der aktuellen Situation wollen wir noch einmal hinweisen auf:

- Henryk Szlajfer, Nachzuholende Entwicklung unter Bedingungen des Weltmarkts: das Beispiel der polnischen Entwicklung, PROKLA 27

- Stefan Horton, Die »Revolution der Hoffnung und ihre Ergebnisse - Einige Bemerkungen zur gegenwärtigen Situation in Polen, PROKLA 27 